\title{
Pentoxifylline Inhibits Transforming Growth Factor-Beta Signaling and Renal Fibrosis in Experimental Crescentic Glomerulonephritis in Rats
}

\author{
Yee-Yung Ng ${ }^{a}$ Yung-Ming Chen ${ }^{b}$ Tun-Jun Tsai ${ }^{b} \quad$ Xiao-Ru Lan ${ }^{c}$ \\ Wu-Chang Yang a Hui Y. Lan ${ }^{c}$ \\ aSection of Nephrology, Department of Medicine, Institute of Clinical Medicine, Taipei Veterans General Hospital, \\ National Yang Ming University, and bepartment of Internal Medicine, National Taiwan University Hospital and \\ National Taiwan University College of Medicine, Taipei, and ' Department of Medicine, University of Hong Kong, \\ Hong Kong, China
}

\section{Key Words}

Pentoxifylline - Crescentic glomerulonephritis •

Transforming growth factor-beta $\cdot$ Epithelial-myofibroblast transition · Smads $\cdot$ Renal fibrosis

\begin{abstract}
Background/Aims: Pentoxifylline (PTX) has been shown to inhibit renal inflammation in a rat model of crescentic glomerulonephritis. The present study investigated the role of PTX in renal fibrosis in rats with crescentic glomerulonephritis. Methods: A rat model of accelerated anti-glomerular basement membrane glomerulonephritis was induced and treated with PTX or vehicle control for 3, 7, 14 and 28 days. The therapeutic effect and mechanism of PTX on renal fibrosis were examined by Northern blot and immunohistochemistry. Results: Diseased rats treated with vehicle control developed a severe crescentic glomerulonephritis with progressive renal fibrosis identified by a marked accumulation of $\alpha$-SMA+ myofibroblasts and collagen matrix. This was associated with tubular epithelial-myofibroblast transition as evident by de novo expression of $\alpha$-SMA and a loss of Ecadherin on damaged tubular epithelial cells. Further studies revealed that severe renal fibrosis was associated with
\end{abstract}

upregulation of renal TGF- $\beta 1$ and activation of TGF- $\beta /$ Smad signaling, which was blocked by treatment with PTX. Conclusions: PTX may be an anti-fibrosis agent capable of inhibiting renal fibrosis in a rat model of crescentic glomerulonephritis. Blockade of TGF- $\beta 1$ expression and Smad2/3 activation may be a mechanism by which PTX inhibits renal fibrosis.

Copyright $\odot 2008$ S. Karger AG, Basel

\section{Introduction}

Upregulation of pro-inflammatory cytokines and adhesion molecules, excessive $\mathrm{T}$ cell and macrophage infiltration, and glomerular crescent formation with rapidly progressive renal dysfunction are the features of crescentic glomerulonephritis. In the past decades, the pathogenic mechanisms of immune-mediated renal injury in crescentic glomerulonephritis have been well elucidated [1]; however, little attention has been paid to the development of renal fibrosis, another critical pathological feature in crescentic glomerulonephritis.

Pentoxifylline (PTX), a clinically available methyl xanthine derivative, is a nonselective phosphodiesterase 
inhibitor with hemorheological activity and anti-inflammatory effects in vivo and in vitro [2]. It has been shown that PTX exerts its anti-inflammatory activities in a number of disease models including anti-GBM crescentic glomerulonephritis [3-6] and human kidney diseases [7-11]. However, it remains unclear whether the therapeutic effects of PTX on kidney diseases are also associated with inhibition of renal fibrosis and what mechanisms may be involved.

In the current study, we found that tubular epithelialmyofibroblast transition (EMT), a critical process in renal fibrosis, occurred in a rat model of anti-GBM crescentic glomerulonephritis. Treatment with PTX was able to block this process and inhibited renal fibrosis. Blockade of renal TGF- $\beta 1$ expression and inactivation of the TGF- $\beta /$ Smad signaling pathway may be a mechanism by which PTX blocks EMT and renal fibrosis in an experimental model of rat crescentic glomerulonephritis.

\section{Subjects and Methods}

\section{Immune Model of Crescentic Glomerulonephritis}

Male Wistar rats (190-200 g) were obtained from the animal center of National Taiwan University Hospital. Experimental crescentic GN was induced as previously reported [5]. Briefly, animals were immunized by subcutaneous injection with $5 \mathrm{mg}$ normal rabbit IgG in Freund's complete adjuvant. Five days later, groups of 6 animals were injected intravenously with $0.5 \mathrm{ml}$ rabbit anti-rat GBM serum $(2.5 \mathrm{ml} / \mathrm{kg}$ body weight, $12.5 \mathrm{mg} \mathrm{IgG} / \mathrm{ml})$, followed immediately by either PTX at a dose of $0.1 \mathrm{~g} / \mathrm{kg} / \mathrm{day}$ (Hoechst, Frankfurt, Germany) or vehicle (phosphate-buffered saline) via intravenous infusion over $1 \mathrm{~h}$ on a daily basis until being killed at days 3, 7, 14 and 28. The experimental protocol was approved by the Animal Experimentation Committee, National Taiwan University.

\section{Renal Histopathology}

Kidney tissues for histological examination were fixed in 10\% formalin and $4-\mu \mathrm{m}$ paraffin sections were stained with hematoxylin and eosin or periodic acid-Schiff reagent (PAS). Glomerular crescent formation and percentage of renal fibrosis in the cortical tubulointerstitium was scored on coded slides as described previously $[5,12]$.

\section{Antibodies}

Antibodies used in this study included: 1A4, which specifically recognized the smooth-muscle $\alpha$-actin isoform in rat, mouse and human (Sigma Chemical Co., St. Louis, Mo., USA); E-cadherin (G-10), an epithelial marker on tubular epithelial cells (Santa Cruz Biotechnology, Calif., USA); TGF- $\beta 1$ and phosphorylated Smad 2/3 (Santa Cruz), and collagen III (Southern Biotech, Birmingham, Ala., USA). In addition, peroxidase-conjugated goat anti-mouse IgG, mouse peroxidase anti-peroxidase complexes, alkaline phosphatase-conjugated goat anti-mouse IgG and mouse alkaline phosphatase anti-alkaline phosphatase complexes (APAAP) were purchased from Dakopatts (Glostrup, Denmark).

\section{Immunohistochemistry}

Double immunohistochemistry was performed using a microwave-based two-color immunostaining technique $[12,13]$. Briefly, sections were dewaxed and treated with microwave oven heating for $10 \mathrm{~min}$ in $10 \mathrm{~mm}$ sodium citrate $\mathrm{pH} 6.0$ to retrieve antigens. Sections were then preincubated for $20 \mathrm{~min}$ in $10 \%$ fetal calf serum to block nonspecific binding, followed by the first primary antibody overnight at $4^{\circ} \mathrm{C}$. After washing and inactivating endogenous peroxidase in $0.3 \% \mathrm{H}_{2} \mathrm{O}_{2}$ in methanol, sections were incubated sequentially with the secondary antibody and then tertiary antibody for $1 \mathrm{~h}$ at room temperature and developed with diaminobenzidine to give a brown product. To block antibody cross-reactivity and inactivate endogenous alkaline phosphatase, sections were treated with microwave again and stained with the second primary antibody overnight at $4^{\circ} \mathrm{C}$. Sections were then incubated with the alkaline phosphatase-conjugated goat antimouse, followed by a mouse APAAP for $1 \mathrm{~h}$ at room temperature, respectively. Finally, sections were developed with Fast Blue BB salt (Sigma) and examined under the microscope.

Semiquantitative Analysis of Immunohistochemical Staining

Total number of cells positive for $\alpha$-SMA and $\mathrm{p}$-Smad $2 / 3$ within the tubulointerstitium were counted under 20 high-power field $(\times 40)$ by means of a $0.02-\mathrm{mm}^{2}$ graticule fitted in the eyepiece of the microscope, and expressed as cells per $\mathrm{mm}^{2}$ as previously described [14]. For tubular epithelial cells that expressed TGF- $\beta 1$ or were transitioning to a mesenchymal phenotype identified as double positive with $\alpha$-SMA and E-cadherin, at least 500 cortical tubules were scored and percent positive for TGF- $\beta 1$ or doublepositive for $\alpha$-SMA and E-cadherin was recorded as previously described [11]. All analyses were processed from the outer to inner cortex, avoiding large vessels and glomeruli. The large vessels, including the arterial walls, were excluded from the study. Because of a diffuse nature in collagen III accumulation in the tubulointerstitium, the percent positive area was semiquantitatively scored as: score $1(<5 \%)$, score $2(5-10 \%)$, score $3(11-15 \%)$, score $4(16-20 \%)$, score $5(21-25 \%)$ and score $6(>25 \%)$. All analyses were performed on coded slides.

Renal RNA Extraction and Northern Blot Analysis

Renal RNA from the cortex was isolated using the guanidinium thiocyanate/acid phenol method and analyzed by Northern blot as previously described [5]. The specific primer pairs for TGF- $\beta 1$ were: upstream, 5'-TCCACAGAGAAGAACTGCTG-3' and downstream, 5'-ACTTGCAGGAGCGCACAATC-3'. The product was subsequently subcloned into the pGEM-dT vector (Promega, Madison, Wisc., USA). The cloned cDNAs were then linearized and used as templates for in vitro transcription of antisense digoxigenin-conjugated riboprobes, following the supplier's instructions (Roche Molecular Biochemicals, Mannheim, Germany).

\section{Statistical Analyses}

Data were expressed as mean \pm SEM. All comparisons were done by one-way analyses of variance with Tukey's comparison program from statistical package for social science (SPSS). $\mathrm{p}<$ 0.05 was considered statistically significant. 


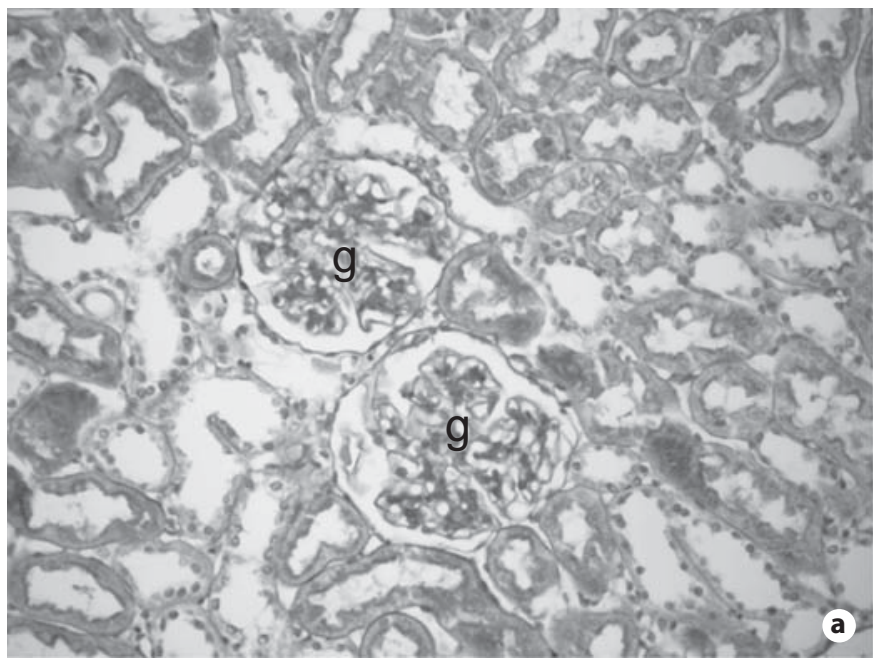

Fig. 1. Effect of PTX treatment on renal histology in a rat model of crescentic glomerulonephritis. a A normal rat kidney. b A diseased rat kidney treated with vehicle control for 28 days. Note that glomeruli are sclerosis (g) and many tubular epithelial cells in the dilated tubules become spindle-shaped in morphology (arrows). c A diseased rat kidney treated with PTX for 28 days. Representative pictures are stained with PAS. $\times 200$.

\section{Results}

\section{Effect of PTX Treatment on Fibrotic Changes in}

Renal Histology

As reported previously [5], rats injected with the antirat GBM antiserum developed severe anti-GBM crescent glomerulonephritis with massive inflammatory/immune response, which is suppressed by PTX. The present study focused on the therapeutic effect of PTX on renal fibrosis. As shown in figure 1, moderate-to-severe glomerulosclerosis and tubulointerstitial fibrosis were developed in diseased animals that had received the vehicle treatment, which is inhibited by treatment with PTX. Interestingly, severe tubulointerstitial fibrosis was associated with a loss of normal tubular epithelial cell morphology, becoming spindle-shaped cells laying the highly dilated tubular
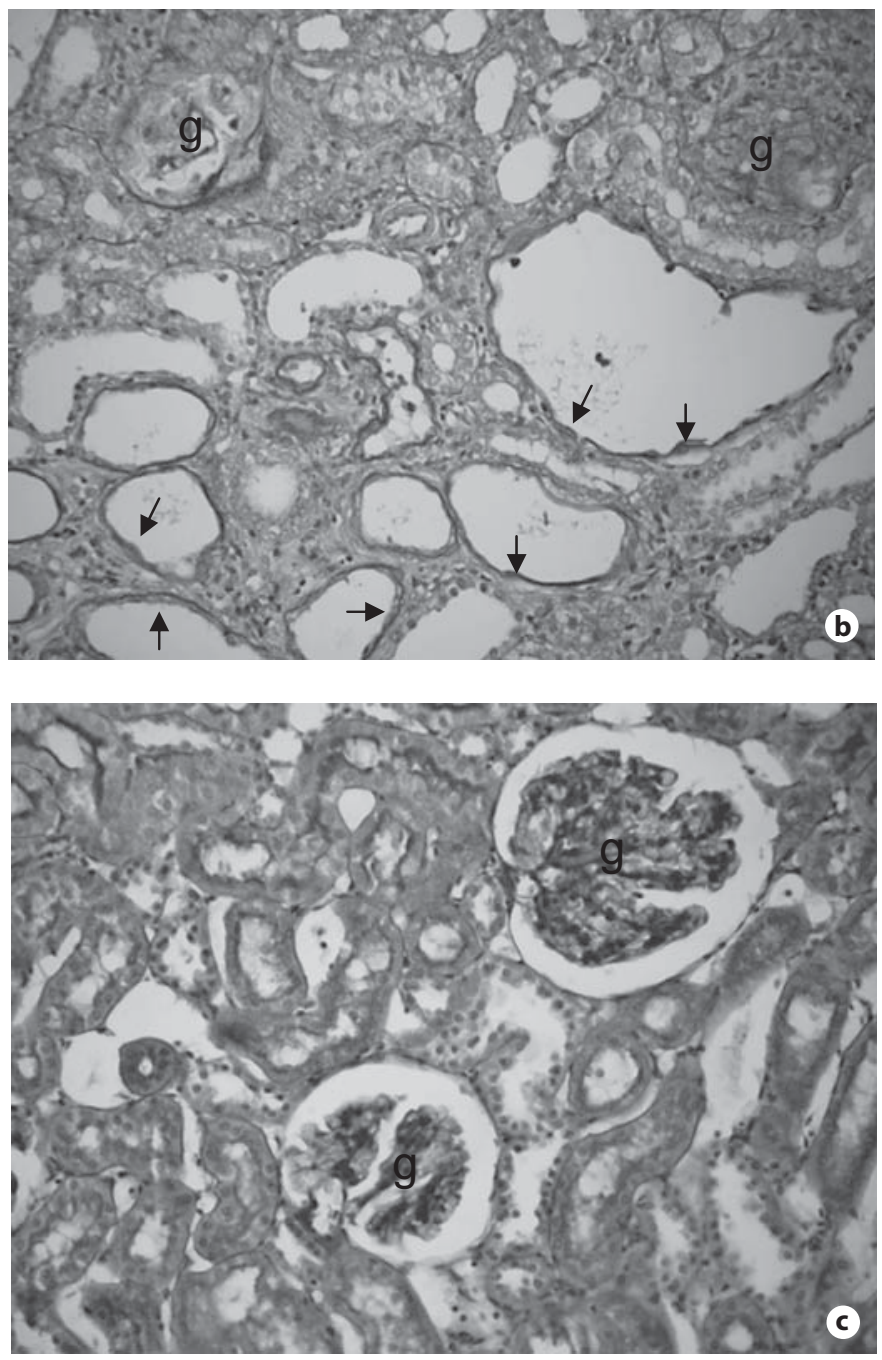

space (fig. 1b), which was also inhibited by PTX treatment (fig. 1c). Immunohistochemically, severe renal fibrosis as identified by a marked accumulation of $\alpha-S M A+$ myofibroblasts and collagen type III was developed in diseased rats treated with vehicle control, which was substantially blocked by the treatment with the PTX (fig. 2a-f). Semiquantitative analysis confirmed these observations (fig. $2 \mathrm{~g}, \mathrm{~h}$ ).

\section{Effect of PTX Treatment on EMT and the \\ Development of Tubulointerstitial Fibrosis in \\ Anti-GBM Glomerulonephritis}

It is now well recognized that EMT plays a role in tubulointerstitial fibrosis [12, 15-17]. Thus, we examined whether EMT occurs in tubulointerstitial fibrosis in the anti-GBM glomerulonephritis model and if PTX treat- 


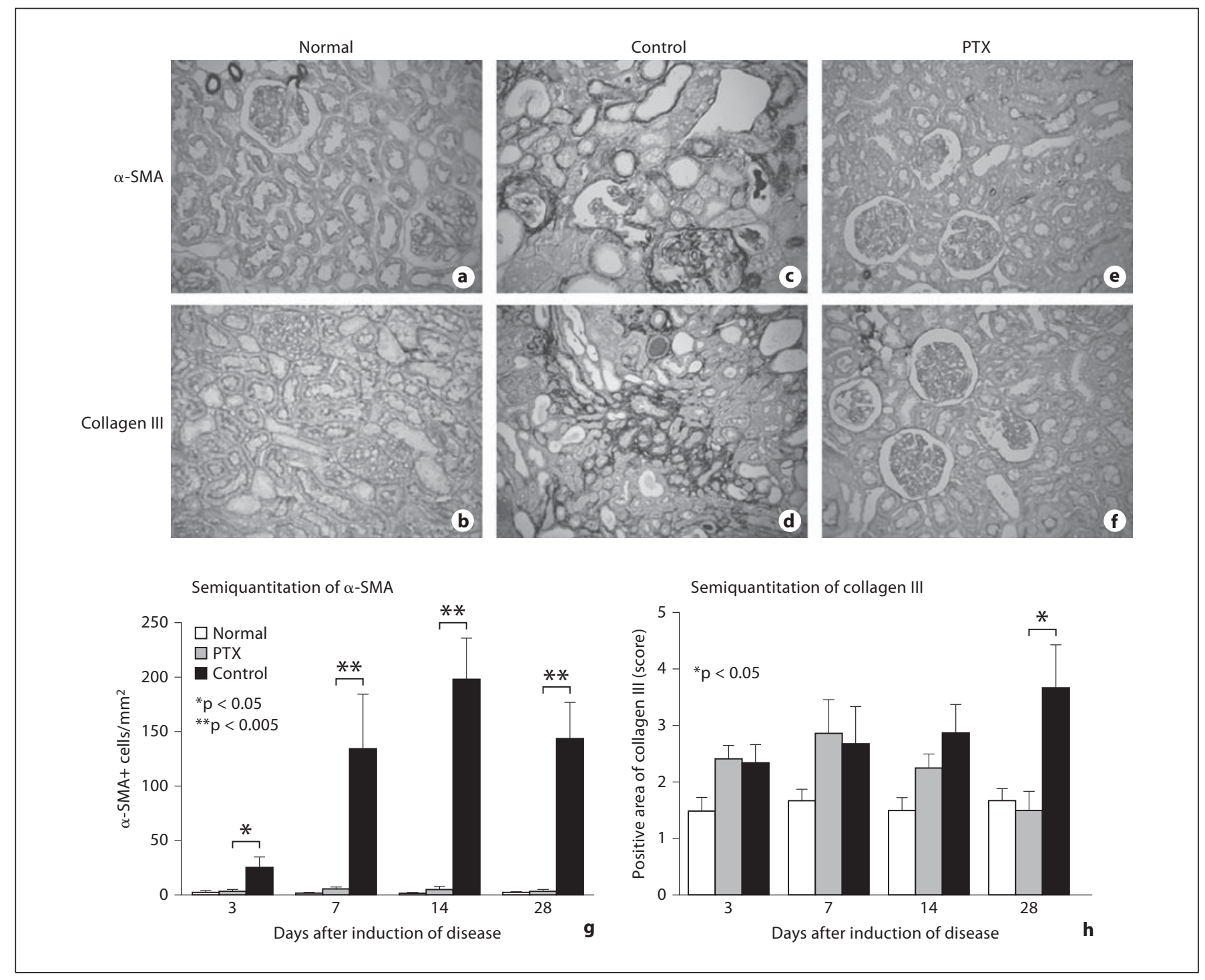

Fig. 2. Effect of PTX treatment on accumulation of $\alpha-S M A+$ myofibroblasts and collagen III in a rat model of crescentic glomerulonephritis at day 28. a, b Normal rat kidney tissues stained with $\alpha$-SMA (a) or collagen III (b). c, d A diseased kidney treated with vehicle control and stained with anti- $\alpha$-SMA (c) or collagen III (d) antibodies. e, $\mathbf{f}$ A diseased kidney with PTX treatment and stain- ing with anti- $\alpha$-SMA (e) or collagen III (f) antibodies. $\mathbf{g}, \mathbf{h}$ Semiquantitative analysis for $\alpha$-SMA+ cells and collagen III accumulation within the tubulointerstitium. Each bar represents the mean \pm SEM for a group of 6 rats. ${ }^{*} \mathrm{p}<0.05,{ }^{* *} \mathrm{p}<0.005$ compared with the PTX treatment. $\times 200$. ment could block this process. As shown in figure 3a, two-color immunohistochemistry revealed that all tubular epithelial cells in normal rat kidneys highly expressed E-cadherin (an epithelial phenotype marker) with complete absence of $\alpha$-SMA (a myofibroblast marker) and tubulointerstitial fibrosis. Strikingly, severe tubulointerstitial fibrosis associated with EMT was observed in vehicle-treated rats (fig. 3b). The transformed tubular epithelial cells showed de novo expression of $\alpha$-SMA with a partial loss of E-cadherin, contributing to focal accumulation of numerous $\alpha$-SMA+ myofibroblasts within the area of severe tubulointerstitial fibrosis (fig. 3b), which was blocked by PTX (fig. 3c). As shown in figure $3 \mathrm{~d}$, semiquantitative analysis showed that a significant EMT occurred at day 7 and this was associated with an increase in interstitial $\alpha$-SMA+ myofibroblast accumulation and the development of collagen matrix accumulation as shown in figures 2 and $3 b$. 

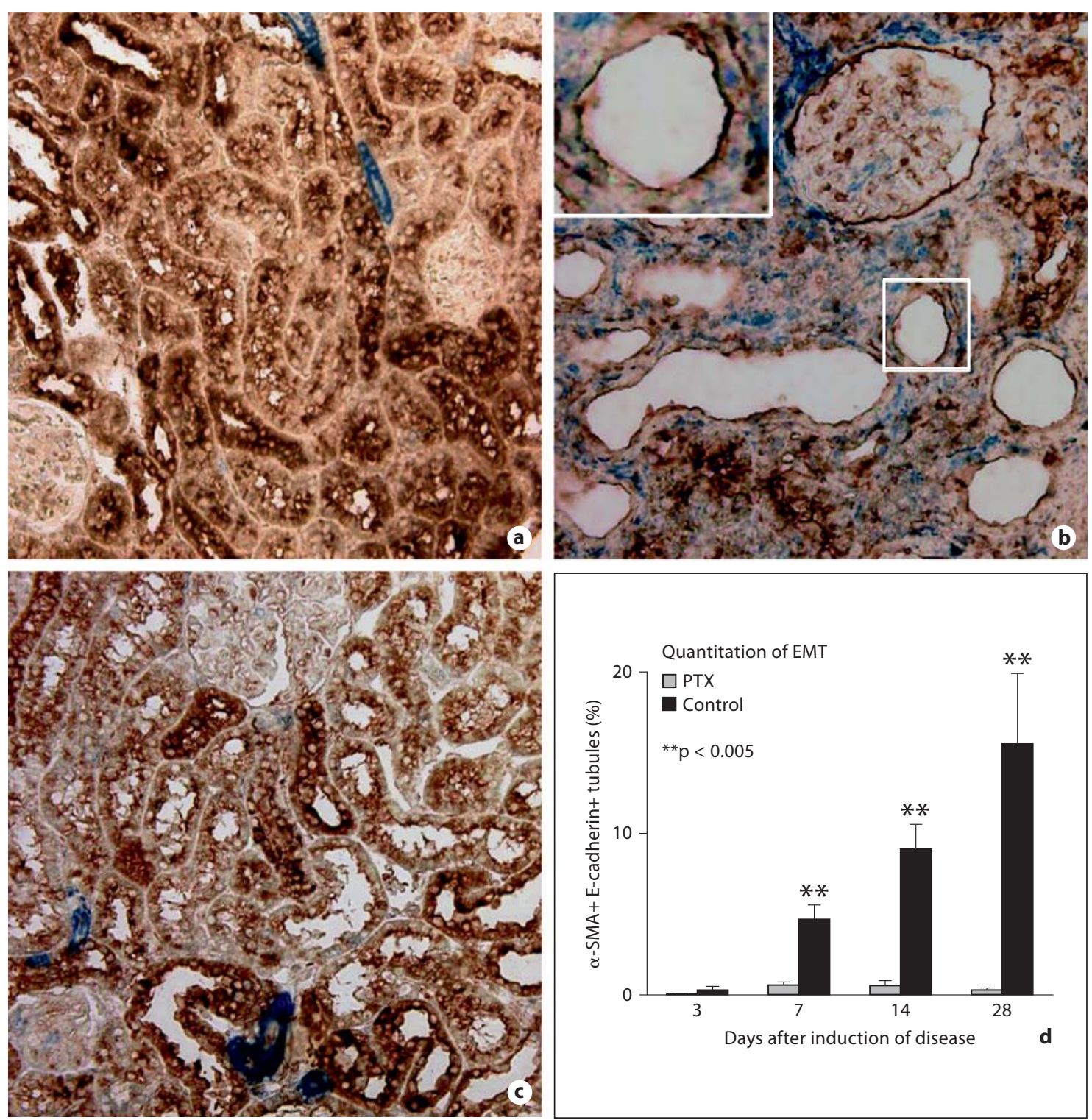

Fig. 3. Two-color immunohistochemical staining shows that treatment with PTX blocks tubular EMT in a rat model of crescentic glomerulonephritis. Brown represents E-cadherin+ tubular epithelial cells and blue represents $\alpha$-SMA myofibroblasts. The mixed color (purple) demonstrates EMT. a A normal rat kidney shows that all E-cadherin+ tubular epithelial cells are absent with $\alpha$-SMA staining. b A control-treated diseased kidney shows that most E-cadherin tubular epithelial cells in the area with massive interstitial $\alpha$-SMA+ myofibroblast accumulation are spindleshaped (EMT) with myofibroblast phenotype (E-cadherin $+\alpha$ $\mathrm{SMA}+$ ), which is further illustrated in the inserted area. c A diseased kidney treated with PTX shows that all E-cadherin+ tubular epithelial cells are absent with $\alpha$-SMA and few $\alpha$-SMA+ myofibroblasts are found in tubulointerstitium. d Semiquantitative analysis. Each bar represents the mean \pm SEM for a group of 6 rats. ${ }^{* *}$ p $<0.005$ compared to the control animals. $\times 200$.
Inhibition of TGF- $\beta$ Expression and Smad2/3

Activation Is a Mechanism by Which PTX Attenuates

Renal Fibrosis in a Rat Model of Anti-GBM

Glomerulonephritis

We next examined the mechanisms whereby PTX inhibits renal fibrosis. Upregulation of renal TGF- $\beta$ and activation of its signaling pathway Smad $2 / 3$ have been shown to play a critical role in renal fibrosis [14]. Thus, expression of renal TGF- $\beta 1$ and activation of $\operatorname{Smad} 2 / 3$ were examined. As shown in figure 4 , Northern blot analysis demonstrated that treatment with PTX showed a significant reduction in renal TGF- $\beta$ mRNA expression 


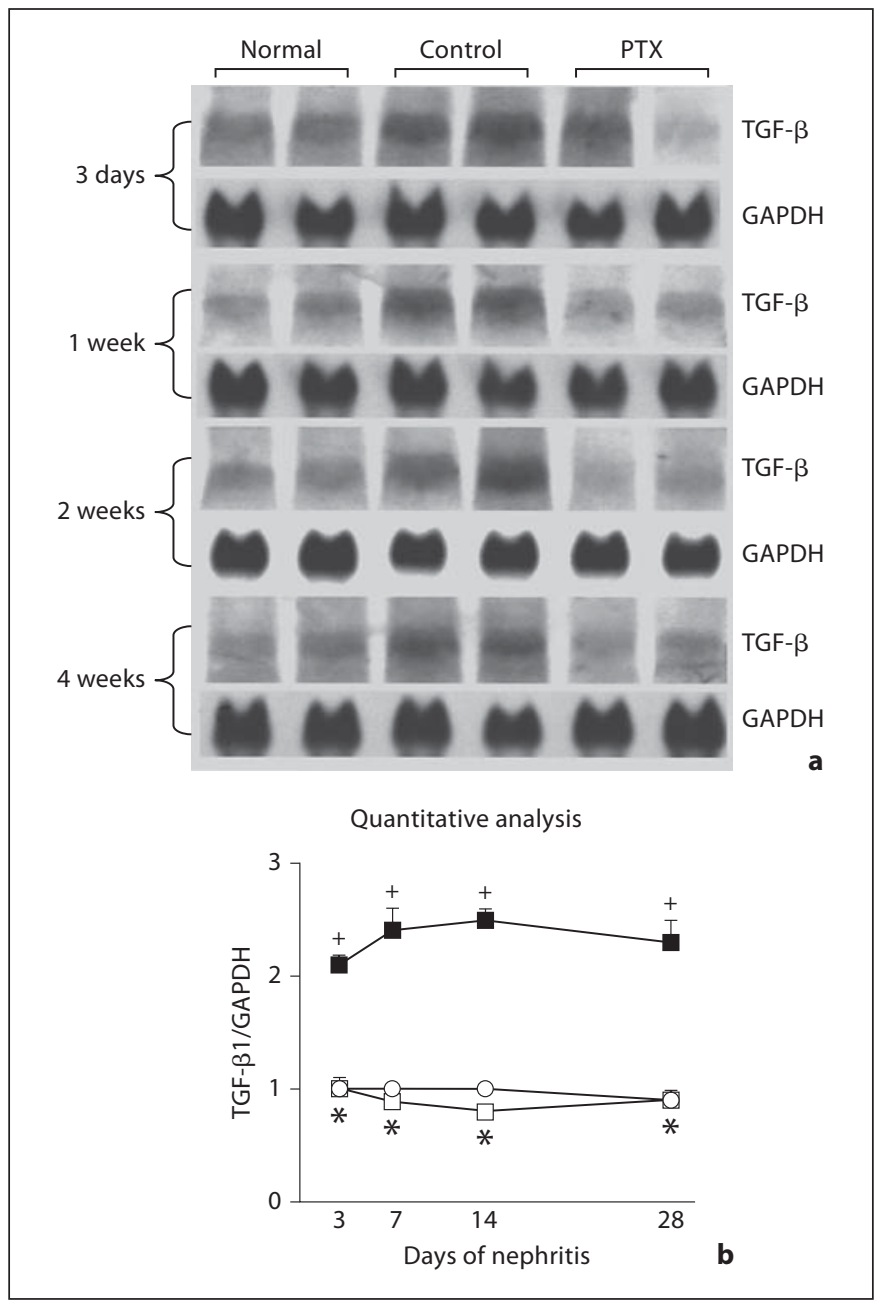

Fig. 4. Northern blot analysis shows that PTX treatment inhibits renal TGF- $\beta 1$ mRNA expression in a rat model of crescentic glomerulonephritis. a Each lane represents one rat kidney and data represents for a group of 6 rats. Representative Northern blots demonstrate that renal TGF- $\beta 1 \mathrm{mRNA}$ is significantly upregulated in control animals, which is blocked in animals treated with PTX. b Quantitative analysis. Each line symbol represents the mean \pm SEM for a group of 6 rats. ${ }^{*} \mathrm{p}<0.05$ PTX-treated vs. vehicle-treated nephritic rats; ${ }^{+} \mathrm{p}<0.05$ vehicle-treated nephritic rats vs normal control rats. $\bigcirc=$ Normal control rats; $\mathbf{\square}=$ vehicletreated nephritic rats; $\square=$ PTX-treated nephritic rats.

from day 3 to day 28 . This was further confirmed by immunostaining with the anti-TGF- $\beta 1$ antibody and we found that upregulation of renal TGF- $\beta 1$ in the diseased kidney of control animals was significantly suppressed by treatment with PTX (fig. 5).

A close association between upregulation of TGF- $\beta 1$ and the development of EMT and tubulointerstitial fibrosis was further examined by two-color immunohisto- chemistry with the anti-TGF- $\beta 1$ and $\alpha$-SMA or collagen III antibodies. As shown in figure 6, upregulation of tubular TGF- $\beta 1$ was closely associated with the de novo expression of $\alpha$-SMA and collagen III by tubular epithelial cells (EMT), contributing to local accumulation of $\alpha$-SMA+ myofibroblasts and collagen III and the development of severe tubulointerstitial fibrosis (fig. $6 a, b$ ), which was inhibited by treatment with PTX (fig. 6c, d).

It is well known that TGF- $\beta 1$ signals through its downstream signaling proteins $\operatorname{Smad} 2 / 3$ to mediate fibrosis. We thus examined the activation of $S \operatorname{mad} 2 / 3$ by their nuclear location using the anti-phosphorylated Smad2/3 antibody. As demonstrated in figure 7, in normal kidney, there was a weak activation of phospho-Smad2/3 in some tubular epithelial cells and glomerular cells as identified by the nuclear pattern of localization (fig.7a, d), indicating a low level of TGF- $\beta$ signaling. In contrast, diseased rats treated with the vehicle control exhibited a marked increase in a nuclear staining pattern of phospho-Smad2/3 in both glomerular and tubulointerstitial areas with severe fibrosis (fig. 7b, d), which was largely inhibited by PTX treatment (fig. 7c, d).

\section{Discussion}

The present study showed that PTX was also an effective agent capable of anti-fibrosis in an immunologically induced glomerulonephritis, in addition to its anti-inflammatory properties as reported previously [2-9]. The anti-fibrotic effect of PTX in a rat model of anti-GBM crescentic glomerulonephritis may be associated with an inhibition of tubular EMT and the production of collagen matrix. Of significance, inhibition of renal TGF- $\beta 1$ expression and $S m a d 2 / 3$ activation may be a critical mechanism by which PTX inhibits renal fibrosis.

Increasing evidence shows that tubular EMT is a mechanism of renal tubulointerstitial fibrosis in both animal models and human chronic kidney disease [12, 1520]. We have previously shown that EMT also participates in the formation and evolution of glomerular crescents in rat anti-GBM glomerulonephritis [21]. In the present study, we further showed that EMT was associated with progressive tubulointerstitial fibrosis in a rat model of anti-GBM glomerulonephritis. Indeed, EMT occurring around day 3-7 was associated with the development of peritubular $\alpha$-SMA myofibroblast accumulation and preceded the development of severe tubulointerstitial fibrosis at day 28, suggesting that EMT is a key process in producing extracellular matrix, leading to an 

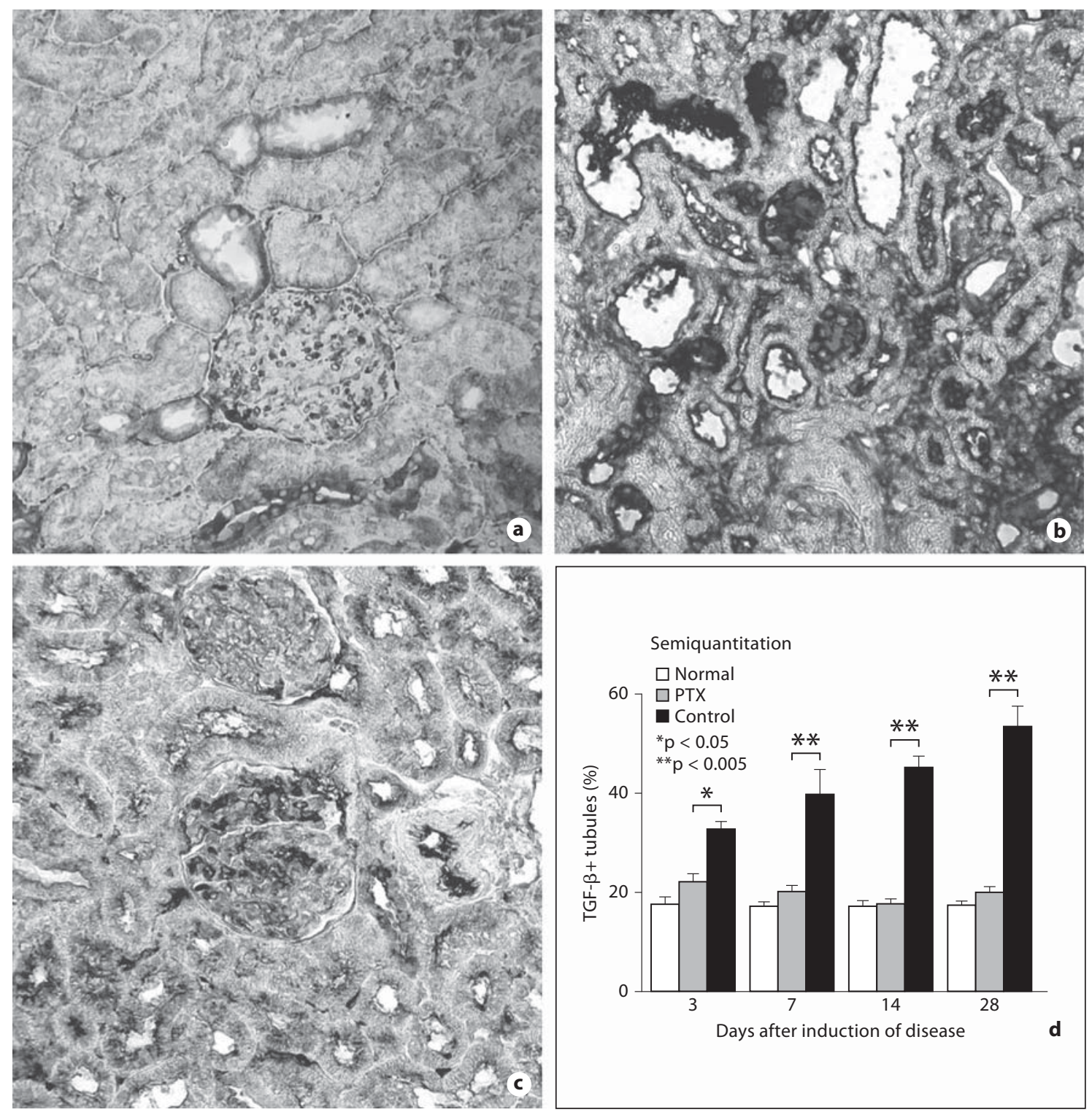

Fig. 5. Immunohistochemistry shows that PTX treatment inhibits renal TGF- $\beta 1$ protein expression in a rat model of crescentic glomerulonephritis. a A normal rat kidney. b A diseased rat kidney treated with vehicle control for 28 days. c A diseased rat kidney treated with PTX for 28 days. d Semiquantitative analysis. Each bar represents the mean \pm SEM for a group of 6 rats. ${ }^{*} \mathrm{p}<0.05$, ${ }^{* *} \mathrm{p}<0.005$ compared with the control animals. $\times 200$. advanced renal fibrosis. Furthermore, consistent with the previous finding that PTX treatment attenuates myofibroblast and collagen matrix accumulation in obstructive kidney disease [22], treatment with PTX was capable of inhibiting the EMT process, thus preventing renal fibrosis. Therefore, results from the present study provided new evidence for the antifibrotic effect of PTX.

It has been shown that PTX is able to inhibit fibroblast growth factor-2-dependent EMT and collagen matrix synthesis in vitro and attenuates tubulointerstitial fibrosis in a rat model of obstructive kidney by blocking Smad3/4-dependent transcription [22,23]. It is now well accepted that TGF- $\beta /$ Smad signaling plays a critical role in the development of renal fibrosis [24]. Inhibition of renal fibrosis in a mouse model of autoimmune glomerulonephritis by a gene transfer of Smad7 demonstrates a role for the TGF- $\beta /$ Smad pathway in the development of renal fibrosis in crescentic glomerulonephritis [25]. In the pres- 

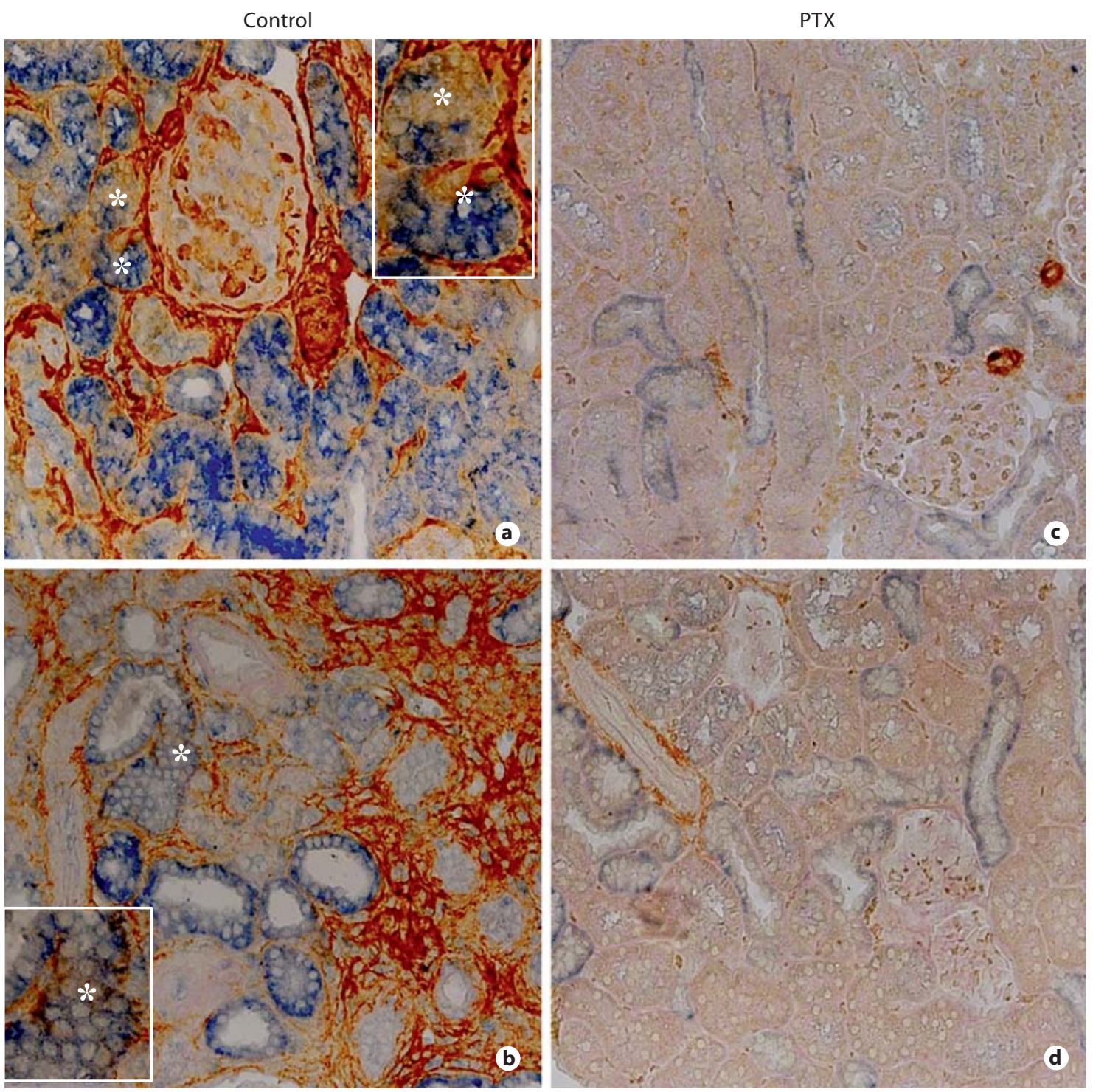

Fig. 6. Two-color immunohistochemical staining shows a close localization of tubular TGF- $\beta 1$ expression and $\alpha$-SMA+ myofibroblast and collagen III accumulation in a rat model of crescentic glomerulonephritis at day 28. Brown represents $\alpha$-SMA+ or collagen III+ staining and blue represents TGF- $\beta 1+$ cells. a A representative diseased kidney treated with vehicle control and stained with $\alpha$-SMA and TGF- $\beta 1$. b A representative diseased kidney treated with vehicle control and stained with collagen III and TGF- $\beta 1$. c A representative diseased kidney treated with PTX and stained with $\alpha$-SMA and TGF- $\beta 1$. $\mathbf{d}$ A representative diseased kidney treated with PTX and stained with collagen III and TGF$\beta 1$. Two-color immunostaining shows that TGF- $\beta 1$ (blue) is strongly expressed by tubular epithelial cells in the area with massive interstitial $\alpha$-SMA+ myofibroblast accumulation (a) and collagen III deposition (b). Note that some tubular epithelial cells with strong TGF- $\beta 1$ expression are also $\alpha$-SMA+ with myofibroblast phenotype $\left(^{*}\right)$ and produce collagen III $\left(^{*}\right)$, which is enlarged in the inserted pictures. In contrast, treatment with PTX blocks TGF- $\beta 1$ expression and accumulation of $\alpha$-SMA+ myofibroblasts and collagen III $(\mathbf{c}, \mathbf{d}) . \times 200$. ent study, we added new data that PTX exhibited its antifibrotic effect on immunologically mediated crescentic glomerulonephritis by suppressing renal TGF- $\beta$ expression and inactivating the downstream of TGF- $\beta$ signaling pathway, Smad2/3. Indeed, marked upregulation of
TGF- $\beta 1 \mathrm{mRNA}$ and protein was evident in the diseased kidney with strong activation of $\operatorname{Smad} 2 / 3$ and severe renal fibrosis. Upregulation of TGF- $\beta 1$ by tubular epithelial cells was associated with EMT as evident by co-expression of TGF- $\beta 1$ and $\alpha$-SMA or collagen III. Treat- 

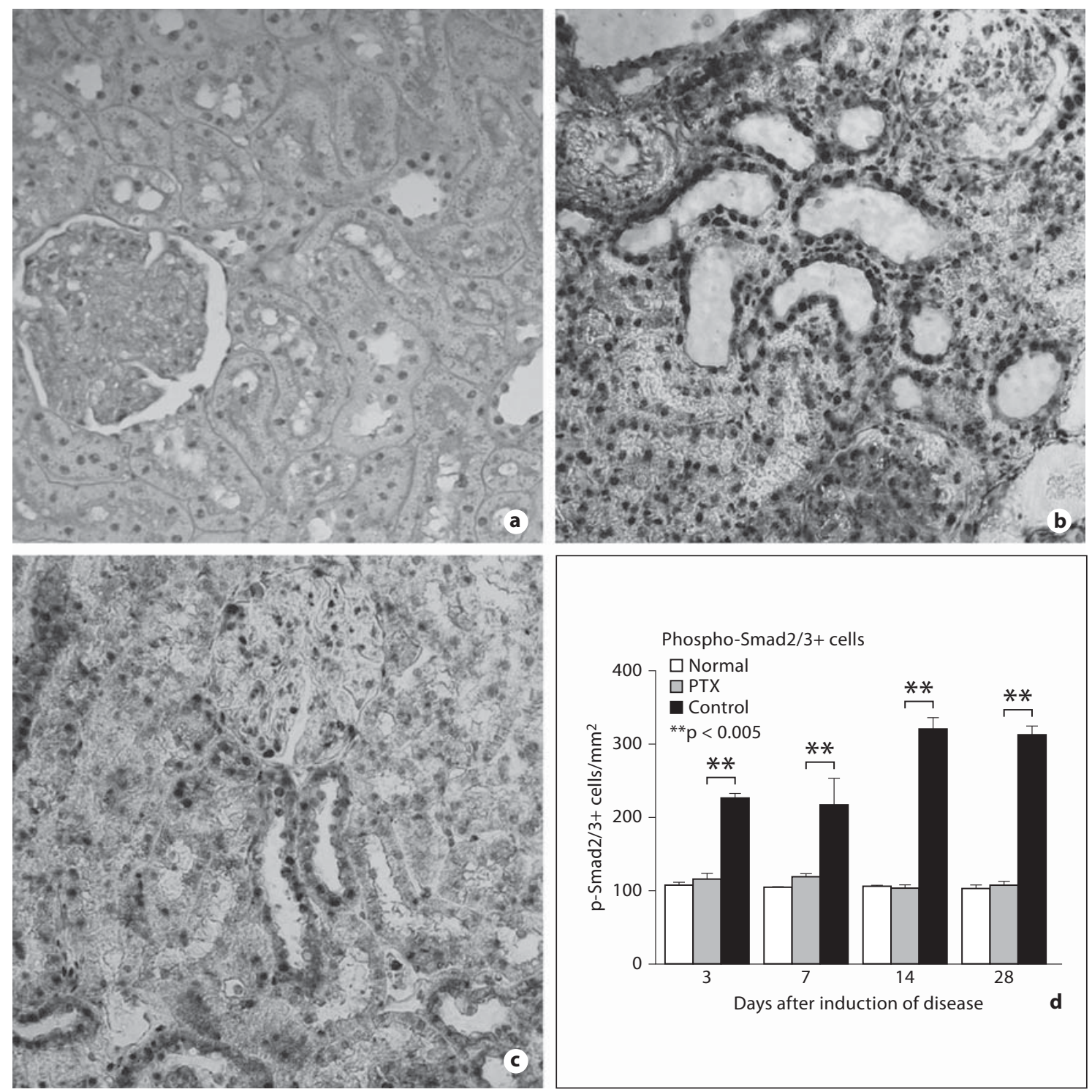

Fig. 7. Immunohistochemistry shows that PTX treatment inhibits Smad $2 / 3$ activation in a rat model of crescentic glomerulonephritis. Activation of TGF- $\beta$ signaling is identified by phosphorylated Smad2/3 nuclear location. a A normal rat kidney. b A diseased rat kidney treated with vehicle control for 28 days. c A diseased rat kidney treated with PTX for 28 days. d Semiquantitative analysis. Each bar represents the mean \pm SEM for a group of 6 rats. ${ }^{* *} \mathrm{p}<$ 0.005 compared with the control animals. $\times 200$. ment with PTX was able to significantly inhibit TGF- $\beta 1$ mRNA and protein expression, thereby preventing EMT and collagen matrix expression. Further study showed that the anti-fibrotic effect of PTX was associated with blockade of the TGF- $\beta 1$ signaling pathway because a marked $S \operatorname{mad} 2 / 3$ activation was significantly inhibited in the diseased kidney treated with PTX. Since TGF- $\beta$ / Smad signaling is a major pathway leading to EMT and renal fibrosis $[15,24,26,27]$, blockade of this pathway is able to suppress TGF- $\beta 1$-induced EMT and renal fibrosis in vivo and in vitro $[14,27]$. Thus, inhibition of TGF- $\beta 1$ dependent $S$ mad $2 / 3$ activation could be a central mechanism by which PTX attenuates renal fibrosis in a rat model of anti-GBM crescentic glomerulonephritis.

It is also possible that inhibition of renal TGF- $\beta 1$ and its downstream mediator CTGF expression by PTX may be a consequence of the anti-inflammatory effect of PTX since this is associated with the anti-inflammatory effect 
of PTX in kidney diseases $[5,9,22]$. Indeed, an increase in pro-inflammatory cytokines (IL-1, TNF $\alpha$ ) within immune-mediated disease kidney is able to stimulate inflammatory cells ( $\mathrm{T}$ cells and macrophages) and resident kidney cells (tubular cells and fibroblasts) to produce growth factors such as TGF- $\beta 1$, which, in turn, can stimulate tubular epithelial cells to undergo the EMT process and activate resident renal fibroblasts to produce extracellular matrix. Our previous finding of IL-1-induced EMT via the TGF- $\beta$-dependent mechanism supports this notion [28]. Once renal inflammation is inhibited, as a result of PTX treatment, upregulation of renal TGF- $\beta 1$ and activation of its downstream Smad signaling pathway could be suppressed. Therefore, PTX may exert its renoprotective effect by blocking both renal inflammation and fibrosis.

\section{Acknowledgements}

This study was supported by grants from the Research Grant Council of Hong Kong (GRF 759206 and 768207), Research and Conference Grant of Hong Kong University (200511159199), and research grant of Taiwan (NSC94-2314-B-075-051).

\section{Conflict of Interest Statement}

The results presented in this paper have not been published previously in whole or part, except in abstract format.

\section{References}

1 Tipping PG, Kitching AR, Cunningham MA, Holdsworth SR: Immunopathogenesis of crescentic glomerulonephritis. Curr Opin Nephrol Hypertens 1999;8:281-286.

$\checkmark 2$ Lin SL, Chen YM, Chiang WC, Tsai TJ, Chen WY: Pentoxifylline: a potential therapy for chronic kidney disease. Nephrology (Carlton) $2004 ; 9: 198-204$.

-3 Chen YM, Chien CT, Hu-Tsai MI, Wu KD, Tsai CC, Wu MS, Tsai TJ: Pentoxifylline attenuates experimental mesangial proliferative glomerulonephritis. Kidney Int 1999;56: 932-943.

-4 Wang W, Zolty E, Falk S, Basava V, Reznikov L, Schrier R: Pentoxifylline protects against endotoxin-induced acute renal failure in mice. Am J Physiol Renal Physiol 2006;291: F1090-F1095.

5 Chen YM, Ng YY, Lin SL, Chiang WC, Lan HY, Tsai TJ: Pentoxifylline suppresses renal tumour necrosis factor-alpha and ameliorates experimental crescentic glomerulonephritis in rats. Nephrol Dial Transplant 2004;19:1106-1115.

6 Lin SL, Chen YM, Chien CT, Chiang WC, Tsai CC, Tsai TJ: Pentoxifylline attenuated the renal disease progression in rats with remnant kidney. J Am Soc Nephrol 2002;13: 2916-2929.

7 Demir E, Paydas S, Balal M, Kurt C, Sertdemir Y, Erken U: Effects of pentoxifylline on the cytokines that may play a role in rejection and resistive index in renal transplant recipients. Transplant Proc 2006;38:28832886.
8 Navarro JF, Mora C, Muros M, Garcia J: Additive antiproteinuric effect of pentoxifylline in patients with type 2 diabetes under angiotensin II receptor blockade: a shortterm, randomized, controlled trial. J Am Soc Nephrol 2005;16:2119-2126.

$\checkmark 9$ Chen YM, Lin SL, Chiang WC, Wu KD, Tsai TJ: Pentoxifylline ameliorates proteinuria through suppression of renal monocyte chemoattractant protein-1 in patients with proteinuric primary glomerular diseases. Kidney Int 2006;69:1410-1415.

-10 Rodriguez-Moran M, Gonzalez-Gonzalez G, Bermudez-Barba MV, Medina de la Garza CE, Tamez-Perez HE, Martinez-Martinez FJ, Guerrero-Romero F: Effects of pentoxifylline on the urinary protein excretion profile of type 2 diabetic patients with microproteinuria: a double-blind, placebo-controlled randomized trial. Clin Nephrol 2006; 66:3-10.

11 Ducloux D, Bresson-Vautrin C, Chalopin J: Use of pentoxifylline in membranous nephropathy. Lancet 2001;357:1672-1673.

12 Ng YY, Huang TP, Yang WC, Chen ZP, Yang $\mathrm{AH}, \mathrm{Mu}$ W, Nikolic-Paterson DJ, Atkins RC, Lan HY: Tubular epithelial-myofibroblast transdifferentiation in progressive tubulointerstitial fibrosis in 5/6 nephrectomized rats. Kid Int 1998;54:864-876.

13 Lan HY, Mu W, Nikolic-Paterson DJ, Atkins RC: A novel, simple, reliable, and sensitive method for multiple immunoenzymed staining: use of microwave oven heating to block antibody crossreactivity and retrieve antigens. J Histochem Cytochem 1995;43:97102.
14 Lan HY, Mu W, Tomita N, Huang XR, Li JH, Zhu HJ, Morishita R, Johnson RJ: Inhibition of renal fibrosis by gene transfer of inducible Smad7 using ultrasound-microbubble system in rat UUO model. J Am Soc Nephrol 2003; 14:1535-1548.

15 Lan HY: Tubular epithelial-myofibroblast transdifferentiation mechanisms in proximal tubule cells. Curr Opin Nephrol Hypertens $2003 ; 12: 25-29$.

16 Liu Y: Epithelial to mesenchymal transition in renal fibrogenesis: pathologic significance, molecular mechanism, and therapeutic intervention. J Am Soc Nephrol 2004;15: 1-12.

17 Kalluri R, Neilson EG: Epithelial-mesenchymal transition and its implications for fibrosis. J Clin Invest 2003;112:1776-1784.

18 Nishitani Y, Iwano M, Yamaguchi Y, Harada K, Nakatani K, Akai Y, Nishino T, Shiiki H, Kanauchi M, Saito Y, Neilson EG: Fibroblastspecific protein 1 is a specific prognostic marker for renal survival in patients with IgAN. Kidney Int 2005;68:1078-1085.

19 Aresu L, Rastaldi MP, Scanziani E, Baily J, Radaelli E, Pregel P, Valenza F: Epithelialmesenchymal transition (EMT) of renal tubular cells in canine glomerulonephritis. Virchows Arch 2007;45:1937-1942.

20 Jinde K, Nikolic-Paterson DJ, Huang XR, Sakai H, Kurokawa K, Atkins RC, Lan HY: Tubular phenotypic change in progressive tubulointerstitial fibrosis in human glomerulonephritis. Am J Kidney Dis 2001;38:761769 . 
$21 \mathrm{Ng}$ YY, Fan JM, Mu W, Nikolic-Paterson DJ, Yang WC, Huang TP, Atkins RC, Lan HY: Glomerular epithelial-myofibroblast transdifferentiation in the evolution of glomerular crescent formation. Nephrol Dial Transplant 1999; 14:1860-1872.

-22 Lin SL, Chen RH, Chen YM, Chiang WC, Lai $\mathrm{CF}, \mathrm{Wu} \mathrm{KD}$, Tsai TJ: Pentoxifylline attenuates tubulointerstitial fibrosis by blocking Smad3/4-activated transcription and profibrogenic effects of connective tissue growth factor. J Am Soc Nephrol 2005;16:27022713.
3 Strutz F, Heeg M, Kochsiek T, Siemers G, Zeisberg M, Müller GA: Effects of pentoxifylline, pentifylline and gamma-interferon on proliferation, differentiation, and matrix synthesis of human renal fibroblasts. Nephrol Dial Transplant 2000;15:15351546.

24 Wang W, Koka V, Lan HY: Transforming growth factor-beta and Smad signalling in kidney diseases. Nephrology (Carlton) 2005; 10:48-56.

25 Ka SM, Huang XR, Lan HY, Tsai PY, Yang SM, Shui HA, Chen A: Smad7 gene therapy ameliorates an autoimmune crescentic glomerulonephritis in mice. J Am Soc Nephrol 2007;18:1777-1788.
26 Fan JM, Ng YY, Hill PA, Nikolic-Paterson DJ, Mu W, Atkins RC, Lan HY: Transforming growth factor-beta regulates tubular epithelial-myofibroblast transdifferentiation in vitro. Kidney Int 1999;56:1455-1467.

27 Li JH, Zhu HJ, Huang XR, Lai KN, Johnson RJ, Lan HY: Smad7 inhibits fibrotic effect of TGF-Beta on renal tubular epithelial cells by blocking Smad 2 activation. J Am Soc Nephrol 2002;13:1464-1472.

-28 Fan JM, Huang XR, Ng YY, Nikolic-Paterson DJ, Mu W, Atkins RC, Lan HY: Interleukin-1 induces tubular epithelial-myofibroblast transdifferentiation through a transforming growth factor-betal-dependent mechanism in vitro. Am J Kidney Dis 2001;37:820-831. 